\title{
High viewership of videos about teenage suicide on YouTube: a cry for help?
}

\author{
Anjali Dagar, M.D. , Tatiana Falcone, M.D. , FAPA \\ Department of Psychiatry/epilepsy, Cleveland Clinic Neurological Institute, Cleveland, $\mathrm{OH}$, USA
}

\begin{abstract}
Introduction
Suicide rate has increased by $31 \%$ and $100 \%$ among boys and girls aged 15 - 19 years, respectively, between 2007 to 2015.

- Social media sites are a major means of interpersonal communication and information gathering for today's teenagers.

YouTube (www.youtube.com), the largest online source of video based digital content on the internet, derives $>70 \%$ users from mobile devices and $95 \%$ of US teenagers have access to smart phones. It is the most commonly used social media platform by teenagers ${ }^{1}$.

Some research has suggested that YouTube may promote the normalization of non-suicidal self-injury among youth ${ }^{2}$ and it may facilitate the rapid spread of harmful trends like the 'tide pod challenge' among teenagers ${ }^{3}$.

- No study, to the best of our knowledge, has investigated the information about suicide among adolescents/teenagers on YouTube and the engagement of viewers with them.
\end{abstract}

\section{Objectives}

In times of rising suicide rate among teenagers, the aim of our study was to analyze the videos on YouTube about this topic, given that they are voracious user of social media.

\section{Methods}

We conducted a comprehensive search on YouTube using a combination of search words like "suicide", "suicidal", "teenager", "teens", "adolescent" etc.

- Unique videos in English language with $\geq 1000$ views were included in final analysis.

- Data on video's view metrics, engagement metrics (number of likes/dislikes), source (uploaded by individuals, academia, activists/media/others) and the type of its content (Table 1) was collected.

- They were categorized and compared based on their source. The tone of top 10 comments, based on viewer engagement, was analyzed as well.

- Statistical analysis, including Kruskal-Wallis test was used to compare the videos.

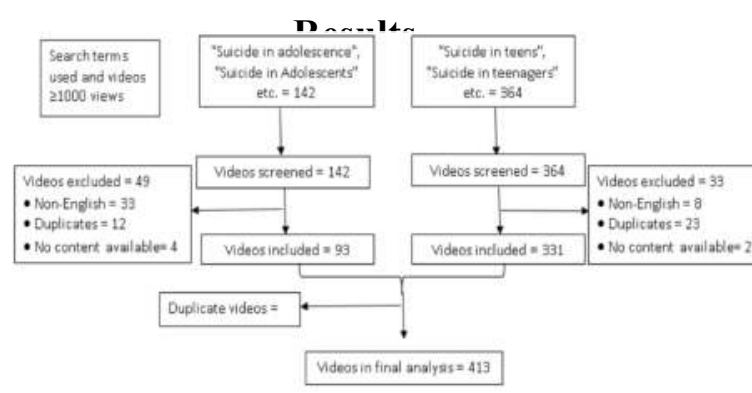

Figure 1. Flowchart of YouTube search and videos included in final analysis

http://www.pewinternet.org/2018/05/31/teens-social-media-technology-2018/ (Accessed on 1/22/2019) Lewis SP1, Heath NL, St Denis JM, Noble R. The scope of nonsuicidal self-injury on YouTube. Pediatrics. $2011 \mathrm{Mar} ; 127(3): \mathrm{e} 552-7$. https://techcrunch.com/2018/01/18/youtube-is-pulling-tide-pod-challenge-videos/ (accessed 5/31/19)

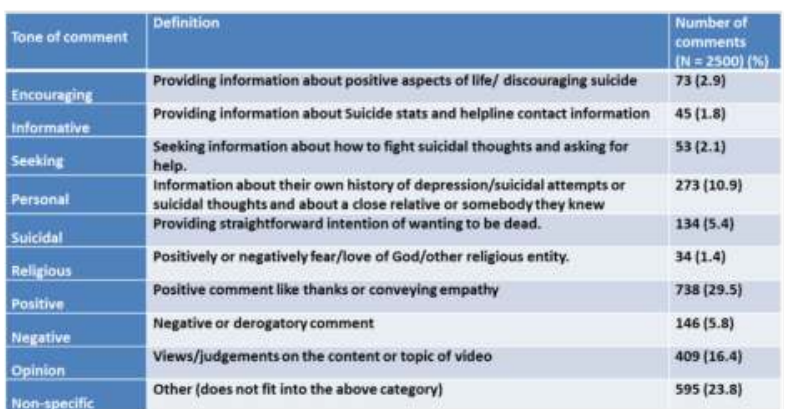

Table 1: Various tones of comments analyzed and their distribution.

A total of 413 videos, with a cumulative $482,736,217$

views, including $24(5.8 \%)$ with a million view each, were analyzed.

Video with highest views (64.9\% of total) was a song titled "1-800-273-8255" (suicide prevention hotline phone number) All, but 22 (5.3\%) videos, mentioned teens, teenagers or adolescents in the video description.

Close to half (48.6\%) videos were educational and almost a third $(29.3 \%)$ about awareness/prevention of teenage suicide.

- Median number of "likes" [45 (14 - 224)] were significantly higher $(\mathrm{p}<0.001)$ compared to the "dislikes" [5 (1 - 19)].

Among the 2500 comments analyzed, a total of $7.5 \%$ expressed frank suicidal ideation or explicitly sought help against such thoughts.

- Only $8.2 \%$ of videos were from academic sources and received significantly lower number of comments compared to video from other sources $(p=0.015)$.

\begin{tabular}{|c|c|c|c|c|c|c|}
\hline Characteristics & & $\begin{array}{l}\text { Total videos } \\
(N=413)\end{array}$ & $\begin{array}{l}\text { Academic (n } \\
\text { =34) }\end{array}$ & $\begin{array}{l}\text { Individuals } \\
(n=121)\end{array}$ & $\begin{array}{l}\text { Activis/M } \\
\text { edis/ } \\
\text { othen (n } \\
\text { ets8) }\end{array}$ & pevalue \\
\hline \multirow[t]{6}{*}{ Vovitube metrics } & View Count & $\begin{array}{l}9860(3007 \\
-35209)\end{array}$ & $\begin{array}{l}4070(1933 \\
-7151)\end{array}$ & $\begin{array}{l}12133(3390 \\
-33633)\end{array}$ & $\begin{array}{l}11105 \\
(2907- \\
37717)\end{array}$ & 0.11 \\
\hline & $\begin{array}{l}\text { Ukes/ Thumbs up, n } \\
\text { (range) }\end{array}$ & $45(14-224)$ & $29(9.67)$ & $\begin{array}{l}61(21- \\
255)\end{array}$ & $\begin{array}{l}42(13 . \\
220)\end{array}$ & 0.11 \\
\hline & $\begin{array}{l}\text { Distikes/ Thumbs } \\
\text { down, n(range) }\end{array}$ & $5(1-19)$ & $2(0-7)$ & $3(1-18)$ & $6(1-21)$ & 0.03 \\
\hline & Days since upload, & $\begin{array}{l}1690 \text { (787 - } \\
2819)\end{array}$ & $\begin{array}{l}1099 \text { (613 - } \\
2906)\end{array}$ & $\begin{array}{l}2404(1125 \\
-3371)\end{array}$ & $\begin{array}{l}1462(661 \\
-2431)\end{array}$ & $\$ 0.001$ \\
\hline & Duration & $\begin{array}{l}3.7(2.2- \\
6.6)\end{array}$ & $4(2.2-5.5)$ & $\begin{array}{l}4.7(3.0- \\
6.1)\end{array}$ & $\begin{array}{l}3.2(2.0- \\
5.2)\end{array}$ & 0.003 \\
\hline & $\begin{array}{l}\text { Number of comments } \\
(n=385)\end{array}$ & $15(3-103)$ & $5(0-28)$ & $23(6-116)$ & $\begin{array}{l}15(3- \\
115)\end{array}$ & $\underline{0.015}$ \\
\hline \multirow[t]{5}{*}{ Type of videe } & Awareness/Prevention & $134(29.36)$ & $9(26.5 \times)$ & $44(36.456)$ & $81(31.4 x)$ & 0.48 \\
\hline & Tribute & $21(6.6 \%)$ & $2(5.9 \%)$ & $9(7.4 \%)$ & $10(3.9 \times 6)$ & 0.29 \\
\hline & Educational & $197(48.6 \%)$ & $18(52.986)$ & $41(33.996)$ & $\begin{array}{l}138 \\
(53.5 \%)\end{array}$ & 0.001 \\
\hline & Advertising & $5(3.96)$ & 0 & $2(1.7 \%)$ & $3(1.26)$ & 0.78 \\
\hline & Personal & 56 (11.6\%) & $5(14.7 \%)$ & $25(20.7 \%)$ & $26(10.1 \times)$ & 0.019 \\
\hline
\end{tabular}

Table 2: Characteristics of videos and user-generated comments

\section{Conclusions}

In contrast to our concerns that led to this study, our findings are quite encouraging:

- $80 \%$ videos were educational/promoted awareness.

- None of the videos encourage teenagers for suicide.

- Video content is overwhelmingly liked by viewers

- One-third comments have positive intonation.

There is a very high viewership for videos on teenage suicide on YouTube, which suggests a yearning for information among youth for this topic.

A small, but significant, number of individuals post their suicidal thoughts or seek help online via their comments. 